\title{
Finding a Simple Polytope from Its Graph in Polynomial Time
}

\author{
Eric J. Friedman
}

Received: 25 September 2007 / Revised: 18 September 2008 / Accepted: 25 October 2008 /

Published online: 19 November 2008

(C) Springer Science+Business Media, LLC 2008

\begin{abstract}
We show that one can compute the combinatorial facets of a simple polytope from its graph in polynomial time. Our proof relies on a primal-dual characterization (by Joswig, Kaibel, and Körner in Israel J. Math. 129:109-118, 2002) and a linear program, with an exponential number of constraints, which can be used to construct the solution and can be solved in polynomial time. We show that this allows one to characterize the face lattice of the polytope, via a simple face recognition algorithm. In addition, we use this linear program to construct several interesting polynomial-time computable sets of graphs which may be of independent interest.
\end{abstract}

Keywords Polytope $\cdot$ Graph · Polynomial time

\section{Introduction}

In [2], Blind and Mani proved, using tools from homology theory, the conjecture of Perles [6] that one can construct the entire face lattice of a simple ${ }^{1}$ polytope from its graph. Then Kalai [6] presented an elegant elementary proof of this result. Whereas Blind and Mani's result was essentially nonconstructive, Kalai's result was constructive but required exponential time to compute (in the size of the graph).

More recently, Joswig, Kaibel, and Körner [3] extended Kalai's analysis to provide polynomial certificates for this problem, based on a pair of combinatorial optimization problems that form a primal-dual pair. However, they did not provide polynomial

\footnotetext{
${ }^{1}$ Note that if the polytope is not simple, then it is not uniquely defined by its graph. Thus, we will only consider simple polytopes.
}

Communicated by Günter M. Ziegler.

E.J. Friedman ( $₫)$

School of Operations Research and Information Engineering, Cornell University, Ithaca, NY, USA

e-mail: ejf27@cornell.edu 
algorithms for either of these problems and thus left open the question of whether this problem can be solved in polynomial time.

In this paper, we present a polynomial-time algorithm for computing the facets of a polytope from its graph, resolving this question. We present a linear program for computing the 2-faces of the polytope from its graph which can be solved in polynomial time. As discussed in [3], this resolves the issue, as it is straightforward to compute the facets of the polytope from the set of 2-faces, in polynomial time. Also, as we show, it is straightforward to characterize the face lattice of the polytope, via a simple face recognition algorithm, i.e., there is a polynomial algorithm for deciding whether a set of vertices form a face of the polytope. ${ }^{1}$

Our discussion in the remainder of the paper will be self contained but compact. See the book [11] for more details on the geometry of polytopes, [3] for more technical details on some of the specific techniques we use, and [4] for an excellent review, including the history, of the problem studied in this paper.

\section{Polytopes and Graphs}

In this paper, we will be concerned with the "combinatorial structure" of a polytope. Thus, we are not concerned with actual realization of the polytope in Euclidean space; however the existence of such an embedding is necessary for the analysis. The following definitions are all standard and are discussed in more detail in standard texts, e.g., [11].

Given a simple ${ }^{2}$ polytope, $P \subset \Re^{d}$, with vertices $V$, we consider its face lattice whose elements are the subsets of $V$ which correspond to faces, where $V_{k}(P)$ is the set of $k$-faces of $P$. For example, the elements of $V=V_{0}$ are all 0 -faces, while the sets of vertices that form facets, $V_{d-1}$, are $(d-1)$-faces. The face lattice is ordered by inclusion.

The graph of the polytope, $G(P)$, is the undirected graph formed by the vertices and their edges (or 1-faces). An orientation on a graph corresponds to a directed version on this graph, and an acyclic orientation is one in which there are no directed cycles.

An abstract objective function (AOF) on $G(P)$ is an acyclic orientation such that the induced directed subgraph on every face of $P$ has a unique sink.

\section{2-Systems}

Now we describe a key lemma. Let $G=(V, E)$ be the graph of a simple polytope, $P$, in $\Re^{d}$, where $V$ is the set of vertices of the polytope, and $E$ are its edges.

\footnotetext{
${ }^{1}$ In [5] an algorithm is presented for computing the entire face lattice in a time that is linear in the size of the face lattice. However, this may be exponential in the size of the initial graph, as there can be exponentially many faces.

${ }^{2}$ A simple polytope is a $d$-dimensional polytope for which every vertex is adjacent to exactly $d$ edges.
} 
A 2-frame, centered at $v$, is a set of three distinct nodes, $\left(v, v^{\prime}, v^{\prime \prime}\right)$ such that $\left(v, v^{\prime}\right) \in E$ and $\left(v, v^{\prime \prime}\right) \in E$. A 2-system is a set of cycles in $G$ such that every 2-frame is contained in a unique cycle.

Let $\mathcal{O}$ be an acyclic orientation on $G$. Define $\mathcal{H}(\mathcal{O})$ to be the number of 2-frames that are sinks under $\mathcal{O}$, where the 2-frame $\left(v, v^{\prime}, v^{\prime \prime}\right)$ is a sink if both edges $\left(v, v^{\prime}\right)$ and $\left(v, v^{\prime \prime}\right)$ are oriented towards the center of the frame, $v$.

Our analysis will be based on the main result from [3].

Theorem 1 (Joswig, Kaibel, and Körner) Let $P$ be a simple d-polytope. For every 2 -system $S$ of $G$ and acyclic orientation $\mathcal{O}$ of $G$, the inequalities

$$
|S| \leq\left|V_{2}(P)\right| \leq \mathcal{H}(\mathcal{O})
$$

hold, where the second holds with equality if and only if $\mathcal{O}$ is an AOF, and the first holds with equality when $S$ is the set of 2-faces.

Thus, if we can find a 2-system $S$ that uniquely maximizes $|S|$ in polynomial time, ${ }^{3}$ then we have found $V_{2}(p)$, and from that it is straightforward to compute facets of $P$ in polynomial time. See [3, 4] for details.

\section{Computing $V_{2}(P)$}

In this section, we will present a binary integer program with an exponential number of variables for computing this 2-system. Somewhat surprisingly, this can be solved in polynomial time.

Let $T$ be the set of all 2-frames in $G$ and $t \in T$ be the 2 -frame $\left(t_{0}, t_{-1}, t_{1}\right)$ centered at $t_{0}$. Let $W$ be the set of cycles in $G$. Then by Theorem 1 in order to compute $\left|V_{2}(P)\right|$ we need to solve

$$
\begin{array}{ll}
\text { (IP-S) } \max \sum_{w \in W} x_{w} \\
\text { s.t. } \quad \forall t \in T, \quad \sum_{w \ni t} x_{w}=1, \\
& x_{w} \in\{0,1\},
\end{array}
$$

where we write $w \ni t$ as a shorthand for the 2-frames $t$ contained in $w$. Note that it is clear from the IP that the maximum will occur with weight only on simple cycles ( since any non-simple cycle can be decomposed into multiple simple cycles, increasing the objective value); however, our analysis will by slightly simplified by allowing non-simple cycles.

\footnotetext{
${ }^{3}$ Note that Joswig, Kaibel, and Körner [3] also show that if $|S|=\left|V_{2}(P)\right|$, then $S=V_{2}(P)$. We do not include it in the statement of the theorem since it follows from the uniqueness of the 2-system that maximizes $|S|$.
} 
First, we consider the following linear relaxation of this integer program:

$$
\begin{aligned}
& \text { (LP-S) } \quad \max \sum_{w \in W} x_{w} \\
& \text { s.t. } \quad \forall t \in T, \quad \sum_{w \ni t} x_{w} \leq 1, \\
& x_{w} \geq 0 .
\end{aligned}
$$

Next, we consider the dual of the linear program

$$
\begin{aligned}
& (\mathrm{LP}-\mathrm{SD}) \quad \min \sum_{t \in T} v_{t} \\
& \text { s.t. } \quad \forall w \in W, \quad \sum_{t \in w} v_{t} \geq 1, \\
& v_{t} \geq 0 .
\end{aligned}
$$

Let IP-SD be the related binary-integer program for LP-SD, i.e., replace $0 \leq v_{t}$ with $v_{t} \in\{0,1\}$.

Now, consider an acyclic orientation $\mathcal{O}$ of $G$ and let $v_{t}=1$ represent the case where the 2 -frame $t$ is a 2 -sink. Then the integer program for minimizing $\mathcal{H}(\mathcal{O})$ over all acyclic orientations can be written by adding to IP-SD the constraint that $v$ must arise from an acyclic orientation on $G$.

$$
\begin{aligned}
(\mathrm{IP}-\mathcal{H}) \quad \min & \sum_{t \in T} v_{t} \\
\text { s.t. } \quad \forall w & \in W, \quad \sum_{t \in w} v_{t} \geq 1, \\
v_{t} & \geq 0, \\
& v_{t} \text { arises from an acyclic orientation of } G .
\end{aligned}
$$

The application of Theorem 1 to this sequence of optimization problems yields the following result:

Theorem 2 Let $P$ be a simple d-polytope with graph $G$. Then the following optimization problems for $G$ all have the same optimal value: IP-S, LP-S, LP-SD, IP-SD, and IP-H.

Proof Let Opt(problem) be the optimal objective value for the optimization problem, "problem." Then it is easy to see that Opt(IP-S $) \leq$ Opt(LP-S) and Opt(LP-SD) $\leq$ $\operatorname{Opt}(\mathrm{IP}-\mathrm{SD}) \leq \mathrm{Opt}(\mathrm{IP}-\mathcal{H})$ as these are sequences of relaxations. By strong duality, we have Opt(LP-S $)=\operatorname{Opt}(\mathrm{LP}-\mathrm{SD})$. Now, Theorem 1 completes the proof since it implies that $\mathrm{Opt}(\mathrm{IP}-\mathrm{S})=\mathrm{Opt}(\mathrm{IP}-\mathcal{H})$.

Thus, we can compute $\left|V_{2}(P)\right|$ by solving either LP-S or LP-SD and in fact compute $V_{2}(P)$ if the optimal solution to LP-S is unique, which we will show below. 


\section{Solving the Linear Program}

Now we show that LP-S can be solved in polynomial time using the ellipsoid algorithm. This is because the dual, LP-SD, has a polynomial number of variables and all constraints have polynomially bounded size (since all the coefficients are 0 or 1 ). Then, because of the equivalence of separation and optimization (see Corollary 14.1g in [9]), all we need to show is that there exists a polynomial-time separation algorithm for LP-SD.

A separation algorithm in this case is an algorithm which, given a vector $v$, can check whether $v$ is feasible and, if not, find a constraint violated by $v$. In our case, such a constraint is a cycle $w \in W$ such that $\sum_{t \in w} v_{t}<1$.

First, we construct the directed graph $G^{T}$ which has a vertex for every ordered 2-frame, e.g., if $\left(v, v^{\prime}, v^{\prime \prime}\right)$ is a frame centered at $v$, then both $\tau=\left(v, v^{\prime}, v^{\prime \prime}\right)$ and $\tau^{\prime}=$ $\left(v, v^{\prime \prime}, v^{\prime}\right)$ are distinct vertices of $G^{T}$. There is a directed edge from $\tau=\left(v, v^{\prime}, v^{\prime \prime}\right)$ to $\tau^{\prime}=\left(w, w^{\prime}, w^{\prime \prime}\right)$ if $v=w^{\prime}$ and $v^{\prime \prime}=w$. If the edge from $\tau$ to $\tau^{\prime}$ exists, then its "cost" is given by $\left(v_{\tau}+v_{\tau^{\prime}}\right) / 2$. It is easy to see that any cycle in this graph has a corresponding cycle in $G(P)$ and the total cost of that cycle is the sum of the $v_{t}$ 's along that cycle, which corresponds to the constraints in LP-SD.

Thus, if the minimum cost cycle in $G_{T}$ has total cost less than 1 , it yields a violated constraint and if it has cost greater than or equal to 1 , then the current solution is feasible. Finding the minimum cost cycle can be done in polynomial time using dynamic programming [1]. A less efficient but easier to describe (and still polynomial-time) algorithm is discussed below.

For every vertex $v$, consider the graph $G_{v}^{T}$ which is constructed from $G_{T}$ by removing all 2-frames which are centered at vertex $v$. Next, consider all pairs of vertices, $\left(\tau, \tau^{\prime}\right)$, where $\tau=\left(v^{\prime}, v, v^{\prime \prime}\right), \tau^{\prime}=\left(w^{\prime}, w^{\prime \prime}, v\right)$, and $v^{\prime} \neq w^{\prime}$. Compute the shortest path from $t$ to $t^{\prime}$ and add the cost of the 2-frame $\left(v, w^{\prime}, v^{\prime}\right)$. The minimum over all such pairs yields the minimum cost cycle that includes vertex $v$. Repeating this procedure for every vertex, allows one to compute the minimum cost cycle for $G_{T}$.

Thus, we have shown the following:

Theorem 3 LP-S is solvable in polynomial time.

\section{Uniqueness of the Optimal Solution}

To complete the analysis one must guarantee that the solution of LP-S is unique.

\section{Theorem 4 LP-S has a unique optimal solution.}

Proof Consider $x^{*}$ which is the incidence vector for the set of 2-faces and note that it is an optimal solution of IP-S. It is also an optimal extreme point solution of LP-S by Theorem 1. In addition, it satisfies the inequalities with equality. Assume that there exists a second optimal extreme point solution of LP-S, $x^{\prime} \neq x^{*}$. Then there must exist some $w \in W$ such that $x_{w}^{*}=1$ and $x_{w}^{\prime}=0$, otherwise $(1+\epsilon) x^{\prime}-\epsilon x^{*}$ would also be an optimal solution for small enough $\epsilon>0$, implying that $x_{w}^{\prime}$ is not an extreme point. 
Let $f \in F$ denote the 2-face implied by $x_{w}^{*}$ and contract the graph $G$ by $f$, denoting this node by $f$ and the contracted graph by $G^{\prime}=C_{f}(G)$. Also let $\mathcal{O}$ be an AOF on $G$ where all edges from $f$ point away from $f$, and let $C_{f}(\mathcal{O})$ be the contraction of this AOF on $C_{f}(G)$. Also define $C_{f}\left(x^{\prime}\right)$ to be the contraction (i.e., projection) of $x^{\prime}$.

Now consider LP-S on $G^{\prime}$ where we ignore the constraints for 2-frames centered at $f$ but require all the remaining ones. Then $C_{f}\left(x^{\prime}\right)$ is feasible for this LP, and its objective value is $\left|V_{2}(P)\right|$. However, the objective value for $C_{f}(\mathcal{O})$ on LP-SD is $\left|V_{2}(P)\right|-1$, which violates weak duality since it cannot be smaller than the objective value for $C_{f}\left(x^{\prime}\right)$ which is $\left|V_{2}(P)\right|$, providing a contradiction and proving the theorem.

Note that LP-SD, IP-SD, and IP- $\mathcal{H}$ all have multiple optimal solutions as a polytope has many different AOFs.

\section{Main Result}

A solution of LP-S, which we can compute in polynomial time, is also the unique optimal solution of IP-S, which yields the set of 2 -faces for $P$. Thus, we have proven our main result.

Theorem 5 One can compute the set of combinatorial 2-faces of a polytope from its graph in time that is polynomial in the size of the graph.

To construct the facets, one needs simply to "grow" them from the 2 -faces. To grow a facet, start with any $(d-1)$-frame centered at $v$ and let $v^{\prime}$ be the unique vertex adjacent to $v$ which is not in that frame. Consider any other vertex $v^{\prime \prime}$ in that frame which is not the center of the frame. Clearly, there exists another frame in the same facet as the first $(d-1)$-frame which is centered at $v^{\prime \prime}$. Now consider the vertex $\hat{v}$ adjacent to $v^{\prime \prime}$ which is contained in the 2-face that contains the frame $\left(v, v^{\prime}, v^{\prime \prime}\right)$. It is straightforward to show that the $(d-1)$-frame centered at $v^{\prime \prime}$ in that facet contains all the vertices except $\hat{v}$. A careful analysis of this procedure is given in [4]. Clearly one can use this procedure to find all the facets in polynomial time.

Corollary 1 One can compute the set of combinatorial facets of a polytope from its graph in time that is polynomial in the size of the graph.

We note that the face lattice may contain exponentially many faces, so exhibiting it explicitly may require exponential time. However, one can implicitly compute the face lattice in polynomial time.

Corollary 2 Let $G=(V, E)$ be the graph of a polytope, $P$, and $W \subset V$. Then one can compute whether $W$ is a (combinatorial) face of $P$ in polynomial time.

Proof By the previous corollary, one can compute the facets of $P$ in polynomial time. It is easy to see that $W$ is a face of $P$ if and only if there exists a set of facets of $P$, 
$F=\left\{F_{1}, \ldots, F_{k}\right\}$ such that $W=\bigcap_{i=1 \ldots k} F_{i}$. This can be computed in polynomial time by letting $F$ be the set of all facets which contain $W$.

\section{Non-Simple Polytopes}

It is tempting to extend our analysis to non-simple graphs. For example, given a graph of a non-simple polytope, $G$, can one find some polytope which has $G$ as its graph. The key impediment in the analysis is the determination of the 2-frames. While every triplet of vertices $v, v^{\prime}, v^{\prime \prime}$ such that $\left(v, v^{\prime}\right)$ and $\left(v, v^{\prime \prime}\right)$ are both elements of $E$ is a 2 -frame in a simple polytope, this is not true in general. For example, when $d=3$, a vertex of degree 4 should only have 42 -frames, not 6 . Thus, prior to solving LP-S, one needs to choose a consistent set of 2-frames. If the choice of 2-frames is correct, then our analysis extends, and one can compute a set of 2-faces (and hence a set of facets) that correspond to some realization polytope.

For a graph that is "mostly simple," that is, a graph for which all but a fixed number of vertices are of degree $d$, and the remaining are of degree $d+k$ for some fixed $k$, one could compute the "2-faces," by solving LP-S for every consistent set of 2-frames. However, finding a correct set of 2-faces is problematic since it is not obviously true that the minimal solution leads to a set of 2 faces for a true polytope, although this is a reasonable conjecture.

\section{AOFs and USOs}

Our analysis suggests that similar techniques might be useful for finding abstract objective functions in polynomial time, an interesting open problem. In addition, these techniques also might be useful for understanding unique sink orientations [10] which drops the requirement that the orientation be acyclic in the integer program IP- $\mathcal{H}$. Also note that IP-SD suggests that a generalization of unique sink orientations which drops the requirement that the sinks arise from an orientation might also be of interest. The relevant parts of Theorem 1 are valid for all of these relaxations of AOFs.

\section{Polynomially Computable Classes of Almost-Polytopal Graphs}

It is known that the problem of checking whether a graph is polytopal is not in co$\mathrm{NP}$ and therefore not in P as discussed in [4]. (In fact, universality theorems [7, 8] suggest, but do not prove, that it is probably at least NP-hard and PSPACE-hard, if not harder.) Thus, it is interesting to find efficiently computable subclasses of graphs which contain the graphs of simple polytopes. Let $\mathcal{G}$ be the set of all graphs and $\mathcal{G}^{\text {SP }}$ be the set of all graphs which arise from simple polytopes.

For example, define $\mathcal{G}^{\mathrm{RC}}$ to be the class of graphs which are $d$-regular and $d$-connected for some $d$. Then, clearly, membership in $\mathcal{G}^{\mathrm{RC}}$ is polynomial computable, and $\mathcal{G}^{\mathrm{SP}} \subset \mathcal{G}^{\mathrm{RC}} \subset \mathcal{G}$, where both inclusions are strict. We are not aware of any significantly tighter classes of graphs in the literature for which membership is polynomial computable. 
However, our linear program provides some new ones. Let $\mathcal{G}^{\mathrm{PD}}$ be the subset of $\mathcal{G}^{\mathrm{RC}}$ for which the linear program (LP-S) has a unique integral solution. One can tighten this class to $\mathcal{G}^{\mathrm{PDF}}$ by using the solution (a set of "2-faces") to construct the facets of the graph using the procedure discussed above and then checking that this set of "facets" is "consistent", e.g., every $(d-1)$-frame is covered exactly once.

Clearly, membership in $\mathcal{G}^{\mathrm{PD}}$ and $\mathcal{G}^{\mathrm{PDF}}$ can be computed in polynomial time, and $\mathcal{G}^{\mathrm{SP}} \subset \mathcal{G}^{\mathrm{PDF}} \subseteq \mathcal{G}^{\mathrm{PD}} \subseteq \mathcal{G}^{\mathrm{RC}} \subset \mathcal{G}$, where we conjecture that all inclusions are strict. However, we do not know the utility of these new classes.

Acknowledgements I would like to thank Adrian Lewis, David Shmoys, Volker Kaibel, Mike Todd, Gunter Ziegler, and 3 anonymous referees for helpful comments and suggestions. This research has been supported in part by the NSF under grant ITR-0325453.

\section{References}

1. Bertsekas, D.P.: Dynamic Programming and Optimal Control. Scientific, Athena (1995)

2. Blind, R., Mani-Levitska, P.: On puzzles and polytope isomorphisms. Aeq. Math. 34, 287-297 (1987)

3. Joswig, M., Kaibel, V., Körner, F.: On the $k$-systems of a simple polytope. Israel J. Math. 129, 109118 (2002)

4. Kaibel, V.: Reconstructing a simple polytope from its graph. In: Jünger, M., Reinelt, G., Rinaldi, G. (eds.) Combinatorial Optimization-Eureka, You Shrink! pp. 105-118. Springer, New York (2002)

5. Kaibel, V., Pfetsch, M.: Computing the face lattice of a polytope from its vertex-facet incidences. Comput. Geom. 23, 281-290 (2002)

6. Kalai, G.: A simple way to tell a simple polytope from its graph. J. Comb. Theory, Ser. A 49, 381-383 (1988)

7. Mnev, N.E.: The universality theorems on the classification problem of configuration varieties and convex polytopes varieties. In: Topology and Geometry-Rohlin Seminar, vol. 1346, pp. 527-543. Springer, Berlin/New York (1988)

8. Richter-Gebert, J., Ziegler, G.M.: Realization spaces of 4-polytopes are universal. Bull. Am. Math. Soc. 32(4), 403-412 (1995)

9. Schrijver, A.: Theory of Linear and Integer Programming. Wiley-Interscience Series in Discrete Mathematics and Optimization. Wiley, Chichester (2000)

10. Szabó, T., Welzl, E.: Unique sink orientations of cubes. In: Proc. 42nd Ann. IEEE Symp. on Foundations of Computer Science (FOCS), pp. 547-555. New York (2001)

11. Ziegler, G.M.: Lectures on Polytopes. Springer, New York (1998) 